\title{
Penetración Dentinaria in vitro del Hipoclorito de Sodio a Diferentes Concentraciones con las Técnicas de Irrigación Convencional y Ultrasónica Pasiva
}

\author{
In vitro Dentinary Penetration of Sodium Hypochlorite at Different Concentrations \\ with the Conventional and Ultrasonic Passive Irrigation Techniques
}

Aurealuz Morales-Guevara

MORALES-GUEVARA, A. Penetración dentinaria in vitro del hipoclorito de sodio a diferentes concentraciones con las técnicas de irrigación convencional y ultrasónica pasiva. Int. J. Odontostomat., 11(3):305-309, 2017.

RESUMEN: El objetivo del presente trabajo fue comparar la penetración dentinaria in vitro entre las concentraciones de hipoclorito de sodio ( $\mathrm{NaClO}$ ) al $5 \%$ y al 2,5\% con técnicas de irrigación convencional e irrigación ultrasónica pasiva. Este fue un estudio transversal, prospectivo y experimental. Se trabajó con 40 segmentos radiculares (especímenes) de $5 \mathrm{~mm}$ de longitud, divididos en 4 grupos. Todos los especímenes se sumergieron en violeta cristal durante 24 horas. Se realizó la preparación biomecánica con sistema rotatorio Pro Taper. Al primer grupo se irrigó con $\mathrm{NaClO}$ al $2,5 \%$ con irrigación convencional, al segundo grupo con $\mathrm{NaClO}$ al 2,5\% con irrigación convencional más irrigación ultrasónica pasiva por 30 segundos, al tercer grupo con $\mathrm{NaClO}$ al $5 \%$ con irrigación convencional y al cuarto grupo con $\mathrm{NaClO}$ al $5 \%$ con irrigación convencional más irrigación ultrasónica pasiva por 30 segundos. Al final de la preparación a todos los especímenes se les realizó un corte mesio distal. La profundidad de la penetración del $\mathrm{NaClO}$ fue deterrminado por el blanqueamiento de la mancha y medido con un microscopio de luz con una magnificación de 40 X. La prueba estadística usada fue ANOVA con un nivel de significancia de $5 \%$. El NaClO al $5 \%$ con técnica de irrigación ultrasónica pasiva presentó la mayor penetración dentinaria in vitro.

PALABRAS CLAVE: dentina, hipoclorito de sodio, irrigación terapéutica.

\section{INTRODUCCIÓN}

El principal objetivo microbiológico de la preparación químico-mecánica de un canal radicular infectado es la eliminación completa de los microorganismos intracanal o la disminución de éstos a niveles compatibles con la cicatrización del tejido perirradicular (Haapasalo et al., 2010). La persistencia de bacterias después de procedimientos químico mecánicos puede influir negativamente en el resultado del tratamiento endodóntico (Cachovan et al., 2013).

Estudios microbiológicos de infecciones endodónticas han demostrado que las bacterias presentes en el sistema de canales radiculares necróticos se pueden encontrar en canales prin- cipales, canales laterales y en los túbulos dentinarios. Una vez que las bacterias invaden los túbulos pueden ser responsables de la persistencia de la infección (Zou et al., 2010).

Estudios anteriores han examinado la profundidad de la penetración de bacterias en la dentina radicular (Kuga et al., 2011; Taschieri et al., 2014; Wong \& Cheung, 2014) y en los túbulos dentinarios (Azim et al., 2016). Las diferentes profundidades de penetración bacteriana pueden estar relacionado con el tamaño de la bacteria, sus características de motilidad, así como diferencias en los diámetros de luz tubular (Zou et al.). 
La acción química del $\mathrm{NaClO}$ juega un rol importante durante la preparación químico mecánica para la remoción de escombros, bacterias, productos tóxicos, y los sustratos necesarios para el crecimiento bacteriano a partir de las superficies inaccesibles, no instrumentadas (Gomes et al., 2009; Vera Rojas et al., 2012; Rossi-Fedele et al., 2013).

El efecto de limpieza del $\mathrm{NaClO}$ se puede mejorar con el uso de irrigación ultrasónica pasiva - PIU- (van der Sluis et al., 2009). El ultrasonido con un irrigante limpia mejor que la irrigación convencional (Muñoz \& Camacho-Cuadra, 2012).

Estudios sobre la eficacia de la irrigación se han centrado en el efecto dentro del canal principal de la raíz (Siqueira et al., 2000; Cachovan et al.). Tener información sobre la penetración del $\mathrm{NaClO}$ dentro de los túbulos de la dentina y los factores que influyen en la profundidad de la penetración sería muy valioso. La concentración del $\mathrm{NaClO}$ y la técnica de irrigación influyen en la penetración de éste en los túbulos dentinarios. El propósito de este estudio fue comparar la penetración dentinaria in vitro entre las concentraciones de $\mathrm{NaClO}$ al $5 \%$ y al $2,5 \%$ con técnicas de irrigación convencional e irrigación ultrasónica pasiva. La hipótesis fue que existen diferencias en la penetración dentinaria in vitro entre las concentraciones de $\mathrm{NaClO}$ al $5 \%$ y al $2,5 \%$ con técnicas de irrigación convencional e irrigación ultrasónica pasiva.

\section{MATERIAL Y MÉTODO}

El presente estudio transversal, prospectivo y experimental fue ejecutado en el Laboratorio de Biología de una Universidad local de la ciudad de Chiclayo- Perú. El tamaño de la muestra se obtuvo teniendo como antecedente la investigación de Zou et al., considerando un nivel de confianza de $95 \%$, potencia de prueba de $80 \%$, varianza de 25 micras, diferencia estimada de 27 micras, obteniéndo como resultado 40 especímenes en total, divididos en 4 grupos de
10. Los criterios de inclsión fueron: premolares humanos monoradiculares, extraídos por motivos ortodóncicos y mantenidos en suero fisiológico, sin reabsorciones ni lesiones cariosas y con canal radicular permeable. Para su ejecución se contó con la aprobación de la escuela de Post Grado de la Universidad Señor de Sipán con Resolución Nº 069-2015/EPUSS-USS

El procedimiento se inició con el retiro de la corona y tercio apical de cada premolar, para obtener especímenes de los tercios cervical y medio, de $5 \mathrm{~mm}$ de longitud. Estos fueron permeabilizados con limas tipo $\mathrm{K}, \mathrm{N}^{\circ} 10$ y 15 , luego se sumergieron en tinte violeta cristal durante 24 horas y se lavaron con agua potable durante 5 minutos. Todo el procedimiento fue realizado por la investigadora principal. Se continúo con la preparación biomecánica usando sistema rotatorio ProTaper® e irrigando con $5 \mathrm{ml}$ de $\mathrm{NaClO}$ entre cada instrumento mediante agujas Navitip ${ }$, al final de la peparación se irrigó con EDTA al 17 $\%$ durante $1 \mathrm{~min}$ siendo el irrigante final siempre el $\mathrm{NaClO}$. El tiempo total de la preparación quimico mecánica fue de $20 \mathrm{~min}$, usándose en total 40 de irrigante en cada especímen. EI NaClO usado fue de la marca comercial Cloromax ${ }^{\circledR} 5$ $\%$, diluyéndose en proporción $1: 1$ con agua potable para obtener la concentración de 2,5\%.

En los grupos que se trabajó con irrigación ultrasónica pasiva se siguió el protocolo de irrigación descrito por los Basrani \& Haapassalo (2012). El equipo de ultrasonido que se utilizó fue de la marca Acteon Satelec P5 Newtron XS®.

Los 4 grupos coformados fueron:

Primer grupo: Se irrigó con $\mathrm{NaClO}$ al 2,5 \% y técnica de irrigación convencional (grupo control). Segundo grupo: Se irrigó con $\mathrm{NaClO}$ al 2,5\% con técnica de irrigación convencional más irrigación ultrasónica pasiva durante 30 segundos. Tercer grupo: Se irrigó con $\mathrm{NaClO}$ al $5 \%$ con técnica de irrigación convencional.

Cuarto grupo: Se irrigó con $\mathrm{NaClO}$ al $5 \%$ con técnica de irrigación convencional más irrigación ultrasónica pasiva durante 30 segundos. 
Al final de la irrigación a todos los especímenes se les realizó un corte mesio distal a lo largo de su eje con discos diamantados a baja velocidad y se procedió a medir con un microscopio de luz Leyca con una magnificación de $40 \mathrm{X}$, obteniéndose fotos en un formato de $2048 \times 1536$ pixeles. La penetración del $\mathrm{NaClO}$ dentro de los túbulos dentinarios se visualizó como una zona desmanchada alrededor de la luz del canal radicular (Fig. 1). La cantidad de desmanche de la dentina se midió en $\mu \mathrm{m}$ en 5 zonas a ambos lados del canal, obteniendo en total 10 mediciones por cada especímen.

Se evaluó la confiabilidad del método con la calibración intraevaluador e interevaluador usando la prueba T- student. Los resultados de las observaciones intra e interevaluador se compararon, no encontrándose diferencias significativas (valores de P entre 0.473- 0.500), por lo tanto las mediciones entre observaciones fueron similares.

El análisis estadístico fue realizado usando el software SPSS 21. La prueba estadística fue ANOVA de un factor, también se utilizó la prueba prueba Post Hoc Bonferroni. El nivel de significancia fue del $5 \%$.

\section{RESULTADOS}

El presente estudio tuvo como objetivo evaluar la penetración dentinaria in vitro del $\mathrm{NaClO}$, para lo cual se conformaron 4 grupos con concentraciones al $5 \%$ y $2,5 \%$ aplicadas mediante las técnicas de irrigación convencional e irrigación ultrasónica pasiva.

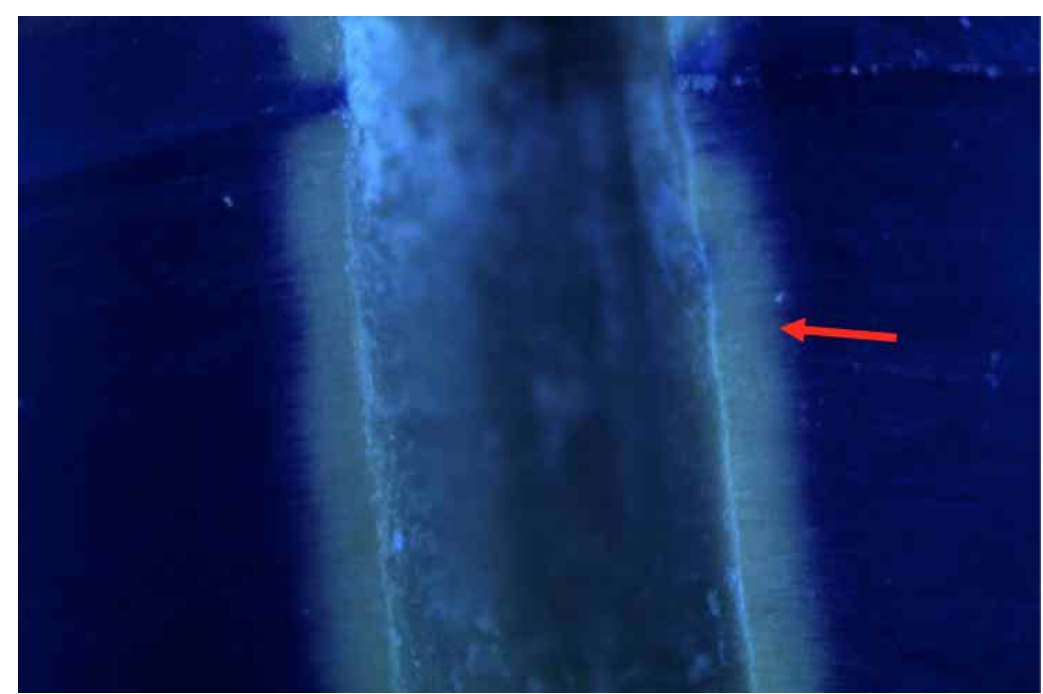

Fig.1. Penetración dentinaria del $\mathrm{NaClO}$ Vista miroscópica con magnificación 40x. Especímen irrigado con $\mathrm{NaClO}$ al $5 \%$ con irrigación convencional más aplicación de ultrasonido durante $30 \mathrm{~s}$.

Se encontraron diferencias estadísticamente significativas entre las concentraciones evaluadas $(p<0.01)$. Se encontró mayor penetración del $\mathrm{NaClO}$ al $5 \%$ que del $\mathrm{NaClO}$ al $2,5 \%$ con irrigación convencional $(p<0,05)$ con medias de $239,80 \mu \mathrm{m}(+/-27.68)$ y $169,20 \mu \mathrm{m}(+/-29.63)$ respectivamente; asimismo, se encontró mayor penetración del $\mathrm{NaClO}$ al $5 \%$ que del $\mathrm{NaClO}$ al 2,5 \% con irrigación covencional más irrigación utrasónica pasiva $(p<0,05)$ con medias de $256,20 \mu \mathrm{m}(+/-39,50)$ y $207,00 \mu \mathrm{m}(+/-38,15)$ respectivamente. Sin embargo, no se encontraron diferencias estadísticamente significativas $(p>0,05)$ entre las técnicas de irrigación evaluadas (Tabla I).

Tabla I. Penetración dentinaria in vitro entre las concentraciones de $\mathrm{NaClO}$ al $5 \%$ y al 2,5\% con técnicas de irrigación convencional e irrigación ultrasónica pasiva

\begin{tabular}{lccccc}
\hline & Media(um) & DE & Min & Max & $P^{*}$ \\
\hline a- NaOCI 2,5\% IC & $169.20^{\mathrm{b}, \mathrm{d}}$ & 29.63 & 129 & 223 & \\
b. $\mathrm{NaOCI} 5 \%$ IC & $239.80^{\mathrm{a}}$ & 27.68 & 189 & 273 & $<0.001$ \\
c. $\mathrm{NaOCl} 2,5 \%$ IC+PUI & $207.00^{\mathrm{d}}$ & 38.15 & 160 & 279 & \\
d. $\mathrm{NaOCI}$ 5\% IC + PUI & $256.20^{\mathrm{a}, \mathrm{c}}$ & 39.50 & 188 & 302 & \\
\hline
\end{tabular}

*ANOVA $(F=12,69)$. Los superíndices indican los grupos con los cuales difirenen estadísticamente (Bonferroni, $p<0,05$ ); IC, Irrigación convencional; PUI, irrigación ultrasónica pasiva; DE, desviación estándar; Min, valor mínimo; Max, valor máximo. 


\section{DISCUSIÓN}

Son varias las variables que influyen en la penetración del $\mathrm{NaClO}$ dentro de los túbulos dentinarios como el tiempo de exposición, el volúmen, la concentración y la técnica de irrigación (Zou et al.); sin embargo, hay controversias sobre el uso de las diferentes concentraciones del $\mathrm{NaClO}$ durante el tratamiento de endodoncia (Radcliffe et al., 2004), además Muñoz \& Camacho-Cuadra afirma que la técnica de irrigación utrasónica pasiva limpia mejor que la irrigación convencional, pero aún no es claro el efecto penetrante del $\mathrm{NaClO}$ en los túbulos dentinarios con esta técnica. En este estudio se analizó la penetración dentinaria empleando $\mathrm{NaClO}$ al $2,5 \%$ y al $5 \%$ con las técnicas de irrigación convencional e irrigación ultrasónica pasiva.

Se analizó dos concentraciones del $\mathrm{NaClO}$ al $2,5 \%$ y $5 \%$, encontrándose que a mayor concentración mayor profundidad de penetración dentinaria. Estos resultados coinciden con los reportados por Wong \& Cheung quienes demostraron que el aumento de la concentración del $\mathrm{NaClO}$ mejora la profundidad de penetración de su acción antibacteriana en los túbulos dentinarios.

No hay muchos estudios que hayan analizado la penetración del $\mathrm{NaClO}$ dentro de los túbulos dentinarios. Los resultados hallados en esta investigación indicaron que al aumentar la concentración del $\mathrm{NaClO}$ del 2,5\% al $5 \%$ se logró una mayor penetración. El valor más bajo de la penetración fue de $129 \mu \mathrm{m}$, valor encontrado en el grupo tratado con $\mathrm{NaClO}$ al 2,5\% con técnica de irrigación convencional y el valor más alto de $302 \mu \mathrm{m}$ fue encontrado en el grupo tratado con $\mathrm{NaClO}$ al $5 \%$ con técnica de irrigación convencional más aplicación de ultrasónido. Estos resultados coinciden con los de Zou et al. quienes reportaron una profundidad de penetración del $\mathrm{NaClO}$ que vario entre 77 a $300 \mu \mathrm{m}$, los parámetros evaluados fueron concentración, tiempo y temperatura.
Actualmente tenemos nuevos dispositivos de irrigación y/o desinfección en endodoncia, entre ellos la Irrigación ultrasónica pasiva, la cual ha mostrado mayor eficacia en la limpieza en zonas de difícil acceso de los canales en comparación con la irrigación convencional (Susin et al., 2010; Castelo-Baz et al., 2012), siendo probable que dicha técnica muestre a su vez una mayor penetración dentinaria del $\mathrm{NaClO}$. En el presente estudio se encontró que a los especímenes que se aplicó irrigación ultrasónica pasiva presentaron una mayor penetración dentinaria del $\mathrm{NaClO}$ en comparación con los especímenes que se irrigaron sólo con la técnica convencional; sin embargo las diferencias no fueron estadísticamente significativas. Tales hallazgos indican que probablemente existen otros factores que influyan en la penetración dentinaria, como es el tiempo de irrigación ultrasónica pasiva. En este trabajo se uso el protocolo de Basrani con un tiempo de irrigación ultrasónica pasiva de 30 segundos; sin embargo, se sugiere la realización de estudios que evalúen un mayor tiempo en el protocolo de irrigación utrasónica pasiva.

Una limitación del presente estudio lo constituye su diseño in vitro; sin embargo, los retos de los estudios in vivo en endodoncia, muchos de los cuales están relacionados con dificultades en la estandarización y el reclutamiento de pacientes, son razones para el esfuerzo por desarrollar modelos experimentales in vitro que refleje la realidad de la situación in vivo.

Los resultados de esta investigación indican que tanto la técnica de irrigación ultrasónica pasiva como la mayor concentración del $\mathrm{NaClO}$ jugaron un rol importante en la determinación de la profundidad de penetración del $\mathrm{NaClO}$ en los túbulos dentinarios. Asimismo, estos resultados permiten reforzar los protocolos clínicos de irrigación que ayudarían a una mayor desinfección del sistema del canal radicular dando las condiciones necesarias al $\mathrm{NaClO}$ para su mayor penetración dentro de los túbulos dentinarios. 


\section{CONCLUSIONES}

El NaClO al $5 \%$ tiene una mayor penetración dentinaria que el $\mathrm{NaClO}$ al $2,5 \%$. Estos resultados sugieren que la penetración dentinaria del $\mathrm{NaClO}$ es mayor con la irrigación ultrasónica pasiva que con la irrigación convencional sola.

AGRADECIMIENTOS. A la Facultad de Ciencias de la Salud y a la escuela de Odontología de la Universidad Católica Santo Toribiio de Mogrovejo por brindar facilidades para el uso del Laboratorio de Biología y equipos.

MORALES-GUEVARA, A. In vitro dentinary penetration of sodium hypochlorite at different concentrations with the conventional and ultrasonic passive irrigation techniques. Int. J. Odontostomat., 11(3):305-309, 2017.

ABSTRACT: The objective of the present study was to compare in vitro dentin penetration between $5 \%$ and $2.5 \%$ sodium hypochlorite $(\mathrm{NaClO})$ concentrations using conventional irrigation and passive ultrasonic irrigation techniques. This was a cross-sectional, prospective and experimental study. It was worked with 40 root segments (specimen) of $5 \mathrm{~mm}$ length, divided into 4 groups. All specimens were immersed in crystal violet for 24 hours. The biomechanical preparation was performed with Pro Taper rotary system. The first group was irrigated with $2.5 \%$ $\mathrm{NaClO}$ with conventional irrigation, the second group with $2.5 \%$ $\mathrm{NaClO}$ with conventional irrigation plus ulrasonic passive irrigation for 30 seconds, the third group with $5 \% \mathrm{NaClO}$ with conventional irrigation and the fourth with $5 \% \mathrm{NaClO}$ with conventional irrigation plus ultrasonic passive irrigation for 30 seconds. At the end of the preparation, all specimens had a distal mesio cut. The depth of $\mathrm{NaClO}$ penetration was determined by bleaching of the stain and measured with a light microscope at a magnification of $40 \mathrm{X}$. The statistical test used was ANOVA with a significance level of $5 \%$. The $5 \% \mathrm{NaClO}$ with passive ultrasonic irrigation showed the highest dentin penetration in vitro.

KEY WORDS: dentin, sodium hypochlorite, therapeutic irrigation.

\section{REFERENCIAS}

Azim, A. A.; Aksel, H.; Zhuang, T.; Mashtare, T.; Babu, J. P. \& Huang, G. T. Efficacy of 4 irrigation protocols in killing bacteria colonized in dentinal tubules examined by a novel confocal laser scanning microscope analysis. J. Endod., 42(6):928-34, 2016.

Basrani, B. \& Haapasalo, M. Update on endodontic irrigating solutions. Endod. Top., 27(1):74-102, 2012.

Cachovan, G.; Schiffner, U.; Altenhof, S.; Guentsch, A.; Pfister, W. \& Eick, S. Comparative antibacterial efficacies of hydrodynamic and ultrasonic irrigation systems in vitro. J. Endod., 39(9):1171-5, 2013.

Castelo-Baz, P.; Martín-Biedma, B.; Cantatore, G.; Ruíz-Piñón, M.; Bahillo, J.; Rivas-Mundiña, B. \& Varela-Patiño, P. In vitro comparison of passive and continuous ultrasonic irrigation in simulated lateral canals of extracted teeth. J. Endod., 38(5):688-91, 2012.

Gomes, B. P.; Martinho, F. C. \& Vianna, M. E. Comparison of $2.5 \%$ sodium hypochlorite and $2 \%$ chlorhexidine gel on oral bacterial lipopolysaccharide reduction from primarily infected root canals. J. Endod., 35(10):1350-3, 2009.

Haapasalo, M.; Shen, Y.; Qian, W. \& Gao, Y. Irrigation in endodontics. Dent. Clin. North Am., 54(2):291-312, 2010.

Kuga, M. C.; Gouveia-Jorge, É.; Tanomaru-Filho, M.; GuerreiroTanomaru, J. M.; Bonetti-Filho, I. \& Faria, G. Penetration into dentin of sodium hypochlorite associated with acid solutions. Oral Surg. Oral Med. Oral Oral Pathol. Oral Radiol. Endod., 112(6):e155-9, 2011.

Muñoz, H. R. \& Camacho-Cuadra, K. In vivo efficacy of three different endodontic irrigation systems for irrigant delivery to working length of mesial canals of mandibular molars. J. Endod., 38(4):445-8, 2012.

Radcliffe, C. E.; Potouridou, L.; Qureshi, R.; Habahbeh, N.; Qualtrough, A.; Worthington, H. \& Drucker, D. B. Antimicrobial activity of varying concentrations of sodium hypochlorite on the endodontic microorganisms Actinomyces israelii, A. naeslundii, Candida albicans and Enterococcus faecalis. Int. Endod. J., 37(7):438-46, 2004.

Rossi-Fedele, G.; Prichard, J. W.; Steier, L. \& de Figueiredo, J. A. The effect of surface tension reduction on the clinical performance of sodium hypochlorite in endodontics. Int. Endod. J., 46(6):492-8, 2013.

Siqueira, J. F. Jr.; Rôças, I. N.; Favieri, A. \& Lima, K. C. Chemomechanical reduction of the bacterial population in the root canal after instrumentation and irrigation with $1 \%, 2.5 \%$, and $5.25 \%$ sodium hypochlorite. J. Endod., 26(6):331-4, 2000.

Susin, L.; Liu, Y.; Yoon, J. C.; Parente, J. M.; Loushine, R. J.; Ricucci, D.; Bryan, T.; Weller, R. N.; Pashley, D. H. \& Tay, F. R. Canal and isthmus debridement efficacies of two irrigant agitation techniques in a closed system. Int. Endod. J., 43(12):1077-90, 2010.

Taschieri, S.; Del Fabbro, M.; Samaranayake, L.; Chang, J. W. \& Corbella, S. Microbial invasion of dentinal tubules: a literature review and a new perspective. J. Investig. Clin Dent., 5(3):163-70, 2014.

van der Sluis, L.; Wu, M. K. \& Wesselink, P. Comparison of 2 flushing methods used during passive ultrasonic irrigation of the root canal. Quintessence Int., 40(10):875-9, 2009.

Vera Rojas, J.; Benavides García, M.; Moreno Silva, E. \& Romero Viñas, M. Conceptos y técnicas actuales en la irrigación endodontica. Endodoncia, 30(1):31-44, 2012.

Wong, D. T. \& Cheung, G. S. Extension of bactericidal effect of sodium hypochlorite into dentinal tubules. J. Endod., 40(6):825-9, 2014.

Zou, L.; Shen, Y.; Li, W. \& Haapasalo, M. Penetration of sodium hypochlorite into dentin. J. Endod., 36(5):793-6, 2010.

Dirección para Correspondencia:

Aurealuz Morales Guevara

Universidad Católica Santo Toribio de Mogrovejo

Chiclayo

PERÚ

E-mail: aurimg@hotmail.com 